\title{
Modeling a subway network: A hot-point attraction-driven evolution mechanism
}

\section{Zhiru Wang}

Lecture, Department of Civil Engineering Management, School of Economics and Management, University of Science and Technology Beijing, No. 30 Xueyuan Road, 100083 Beijing, China. Tel.\& Fax: 86106233 3254; Visiting Scholar, Department of Information Systems and Emergency Management School of Business and Economics, Loughborough University, Loughborough LE11 3TU, United Kingdom. E-mail: wangzhiru@ustb.edu.cn

\section{Fangyan Niu}

Undergraduate student, Department of Civil Engineering Management, School of Economics and Management, University of Science and Technology Beijing, No. 30 Xueyuan Road, 100083 Beijing, China. E-mail: 13121999855@163.com

\section{Lili Yang}

Reader in Department of Information Systems and Emergency Management, Management School of Business and Economics, Loughborough University, Loughborough LE11 3TU, United Kingdom. E-mail: L.Yang@Iboro.ac.uk

\section{Guofeng Su}

Professor, Centre for Public Safety Research, Tsinghua University, No. 30, Shuangqing Road, 100084 Beijing, China. E-mail: sugf@tsinghua.edu.cn

\section{Abstract}

This study proposes a hot-point attraction (HPA)-driven model considering both transfer station attraction and location centrality attraction and accompanies a random walk mechanism in 
a specified direction to meet the gap of the Barabási-Albert (BA) model in subway network evolution. A comparative analysis between BA model and HPA-driven evolution model is performed via simulation experiment. The results show that the topological characteristics and average probability of access distance obtained by the HPA are more in agreement with the real subway network. It is expected that the results of the study will more realistically reflect the evolutionary mechanism of subway networks and provide the basis for decisions on subway network structures in the design stage and network dynamics in resilience governance.

Keywords: Subway network, Evolution mechanism, Geographic location attraction, Complex network theory, Scale-free network, Network Modeling.

\section{Introduction}

A subway system consists of multiple subsystems that interact and depend on each other. The subway topology network corresponds to the underlying network that interacts between subsystems. In the network, each node denotes a station, and the section between stations is denoted as the edge in the network (L-space) [1-3]. The connections between stations in the subway network are typically complicated, and the functions and dynamics of the subway system are closely related to the topology of the subway network [4]. In order to understand and perform an in-depth investigation of the topology characteristics and dynamic complexity of a subway system, it is necessary to establish a reliable network evolution model to explain specific statistical properties of the actual network [5]. Extant studies proposed a variety of public transport network 
evolution models including the random connection model of a subway [6], spatial evolution model of public transport [7], R-space OD-driven evolution model [8], network operator game model and other traffic domain fitness models [9], attractiveness model [10], and Caveman model [11]. Previous studies on the models provide the necessary technical means to examine subway network systems.

In a subway network, a few stations are connected by multiple lines, and this implies that the preference of the topology affects the evolution of the network [12]. Several researchers performed an empirical analysis to obtain the distribution of the power distribution of the metro network obeying the power law distribution $[2,5,7-8,13-15]$. Topological preference significantly affects the evolution of the network. However, empirical studies indicate that it is not the only mechanism that governs the evolution of the subway network, and its degree distribution does not strictly obey the power law distribution or exponential distribution [14, 16-17]. Specifically, in addition to topological preferences, for the aforementioned types of spatial networks, geographic factors typically significantly affect topology and its evolution [18]. While planning a new subway line, in order to ensure the fairness and efficiency of travel [19], new lines are more inclined to connect with hot-point which attracts a lot of people, like a city's commercial activity centers, political activity centers, and tourist attractions [20].

While implementing the topological preference attachment mechanism, a feasible method involves considering a station's transfer ability [9]. It is generally believed that stations with high 
transfer capabilities tend to exhibit high degrees of value [21, 22]. However, in subway systems, most of the stations include only one line that passes through, and only a few stations include several lines passing through. Hence, the probability of selecting the station with a high line degree, i.e., the BA model, is low. The essence of the problem is explained from the viewpoint of mathematical analysis. When the denominator of the mathematical analytic is high, the magnitude of the probability does not depend on the numerator, which is considered by [14] as a large network evolution with new line but not a station. The discussion of node selection probability and node degree is consistent. The probability that the BA model is selected to the rich node is still small, and the preferred probability model needs to be improved. Therefore, some adaptation may be required [23].

There are two main points in the limitation of geographical factors on the formation of networks, namely the probability of connecting edges between nodes to decay with power and exponential $[24,25]$. With respect to the subway network, it denotes the transfer capacity, centrality, and geographic location of the station. It should be noted that a few extant studies suggested that each node exhibits its own range of perception [16,24-25]. Nodes can only connect with nodes within their perceived range, and the sensing range of different nodes is different. This idea corresponds to node fitness, and geographical factors are subtly combined.

Furthermore, an extant study performed empirical research and indicated that the degree distribution of the subway network obeys the shifted power law (SPL) distribution [26], i.e., the 
evolution of the subway network exhibits topological and geographical preference attachment characteristics as well as randomly connected features. The empirical result cannot be explained by existing evolutionary models. The growth of the subway network is based on physical lines [14, 26-27], and their nodes exhibit exact geographical locations, and their joint edges are also subject to geographical factors [22]. Subway networks, aviation networks, and railway networks correspond to typical examples of the aforementioned types of space networks. Stations on new lines tend to connect to old stations that are closer to their location since building long distances typically entails significant cost and expense [28].

The Ref. [14] used L-space modeling to establish a subway evolution model. While generating a network, the probability of all old stations being selected is identical and is independent of the number of lines passed by the station. In the simulation method, the station degree obeys the exponential distribution. To improve the selection mechanism, the Ref. [29] assumes that the number of stations in each new line follows the normal distribution. They used the P-space method to construct mathematical models and analyzed the simulated network eigenvalue changes in three situations where the proportion of new lines with old stations corresponds to $0.1,0.6$, and 0.9 . in Ref. [30] adjusted the global coupled community connection of the BA model to the local coupled community connection model. However, the new line selected the old station with random probability $\mathrm{P}$, and the heterogeneity of the resulting subway network was significantly correlated with probability P. The aforementioned studies improved the node as the growth unit of network to the line as the growth unit. Nevertheless, the model does not consider the attraction of the transfer station to the new line in the subway network, and the 
centrality of the station $[16,31,32]$.

The modeling studies of spatial networks provide a few ideas to model subway networks. Based on the aforementioned studies, the present study proposes a subway network evolution model based on the hot-point of the city to attract new lines. The subway station corresponds to the node in the network, and the connecting edge between the nodes corresponds to the physical track between the stations. During the formation of the subway network, the network was expanded by the addition of new lines. From the perspective of passenger travel convenience, the new route is preferred to transfer stations or stations located in the center of the city, transportation centers, political activity centers, and tourist attractions as access points for new lines. Stations on new line walk randomly in the traffic corridor, the phenomenon is consistent with the empirical research results [26]. The study proposes the attraction model of the new line via the city's hot point, both considers the attraction of the transfer station to the new line and centrality of the station location to attract the new line. Further comparative analysis of a real subway network as established by BA model and our HPA-driven evolution model is performed via a simulation study.

\section{Exploring static properties}

We focus on the evolution of subway topological structure. Thus, we consider four typical subway networks which exist in major cities of the world. These include Beijing, Shanghai, Tokyo, and Moscow, and a sample is shown in Fig. 1. Additionally, we focus on urban subway systems and do not consider longer-distance heavy and light-rail commuting systems in urban areas. 

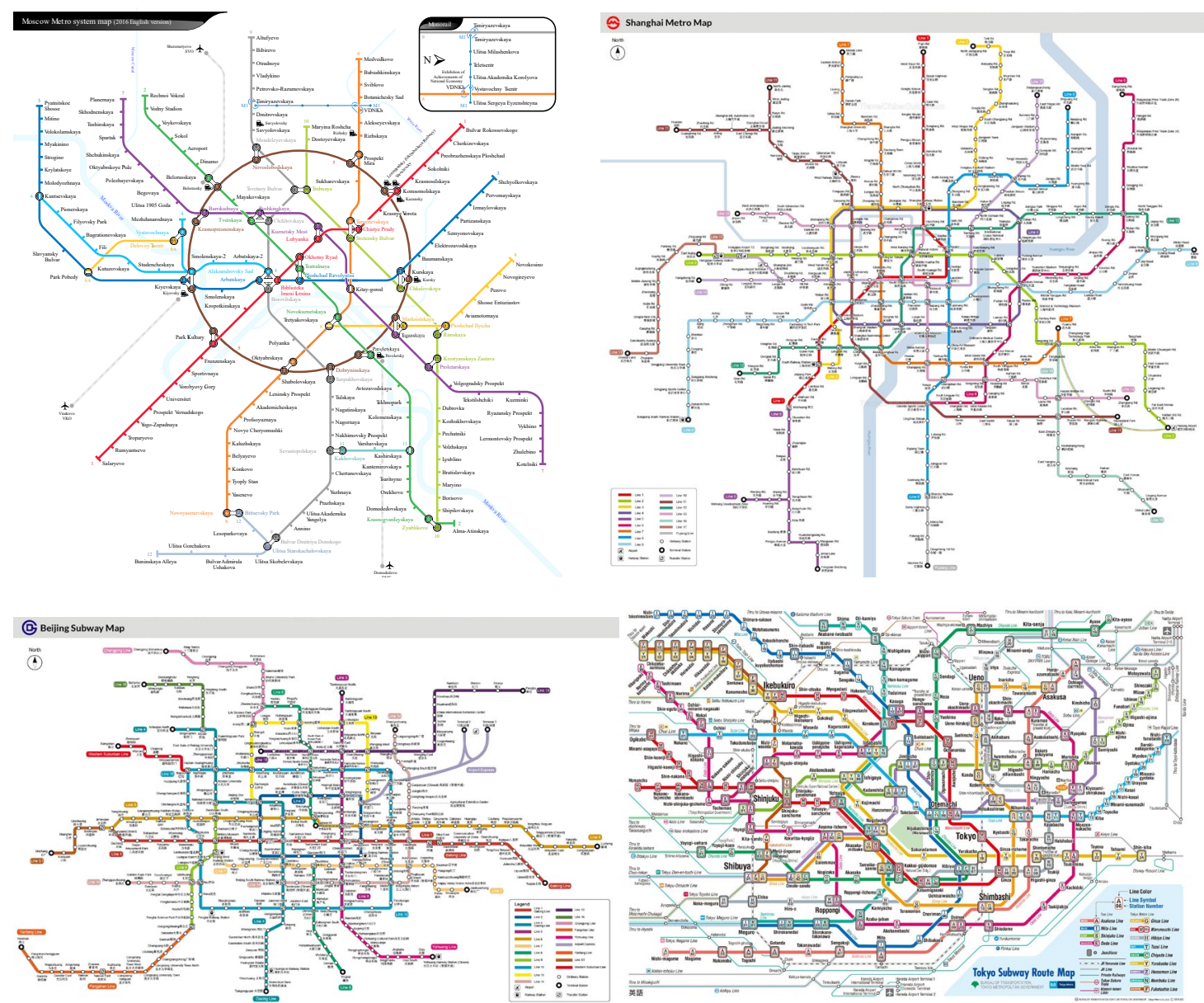

Fig. 1 Topological structure of the subway network. From the left to right and top to bottom:

Shanghai, Moscow, Tokyo, and Beijing (Figures from the official website of subway corporation).

The network topology was constructed using two main data sources. First, current network maps produced in 2018 were used to define lines for each network and then to define line-based topological preference, namely which station follows another station on each line. The second type of data was gathered from official subway websites. For most networks, there is one page per station with various information including the first date of operation, precise location and address, and number of passengers.

Static properties of transportation networks were examined for several years. The studies 
revealed a few significant similarities between different networks despite differences in their historical development and in the cultures and economics in which they were developed. In Ref. [26], SPL was observed for 52 world subway networks. This indicated that although the topological preference significantly affects the evolution of the network, empirical results indicate that it is not the only mechanism that governs the evolution of the subway network.

Based on Vuchic's public transport planning theory [16], while planning a new subway line, the new line is more likely to connect to the city's hot point to ensure fairness and efficiency. The transportation hub, business activity center, political activity center, and tourist attractions are always associated with the hot points in the city that attract resumes, restaurants, individuals, and transport resources. In a city, particularly a megacity, there will be more than one hot points, although they are in different locations. For example, in Beijing, the business activity center is in the Chaoyang district and the political activity center is in the West City district, although the transportation hub and tourist attractions are scattered in the six administrative regions. The same phenomenon is observed in other megacities such as Shanghai and Guangzhou. Therefore, we assume that the barycenter of each operation line corresponds to the attraction hub. Increasing the closeness of a station to the barycenter adds its attraction to new lines. Therefore, the attraction capacity of the station is defined as its HPA ability that is quantitatively assessed by the distance from the barycenter station

The distance from the access station to barycenter is counted manually. The section number between the access station and barycenter is the distance. The proportion of a certain distance of 
the preferred access station is calculated to determine whether there is a rule that new lines preference to the stations with the higher ability of HPA by an empirical static property analysis. The calculation result is shown in Fig. 2. The horizontal axis denotes the distance of the preferred station from the barycenter of the line. Decreasing the distance reduces the centrality of the station. For example, distance 0 implies that the barycenter station corresponds to the preferred station of the new line. Distance 15 implies that there are 15 stations between barycenter stations to the preferred station. The vertical axis indicates the probability that a preferred station with a certain centrality is preferentially selected as an access station for the new line.

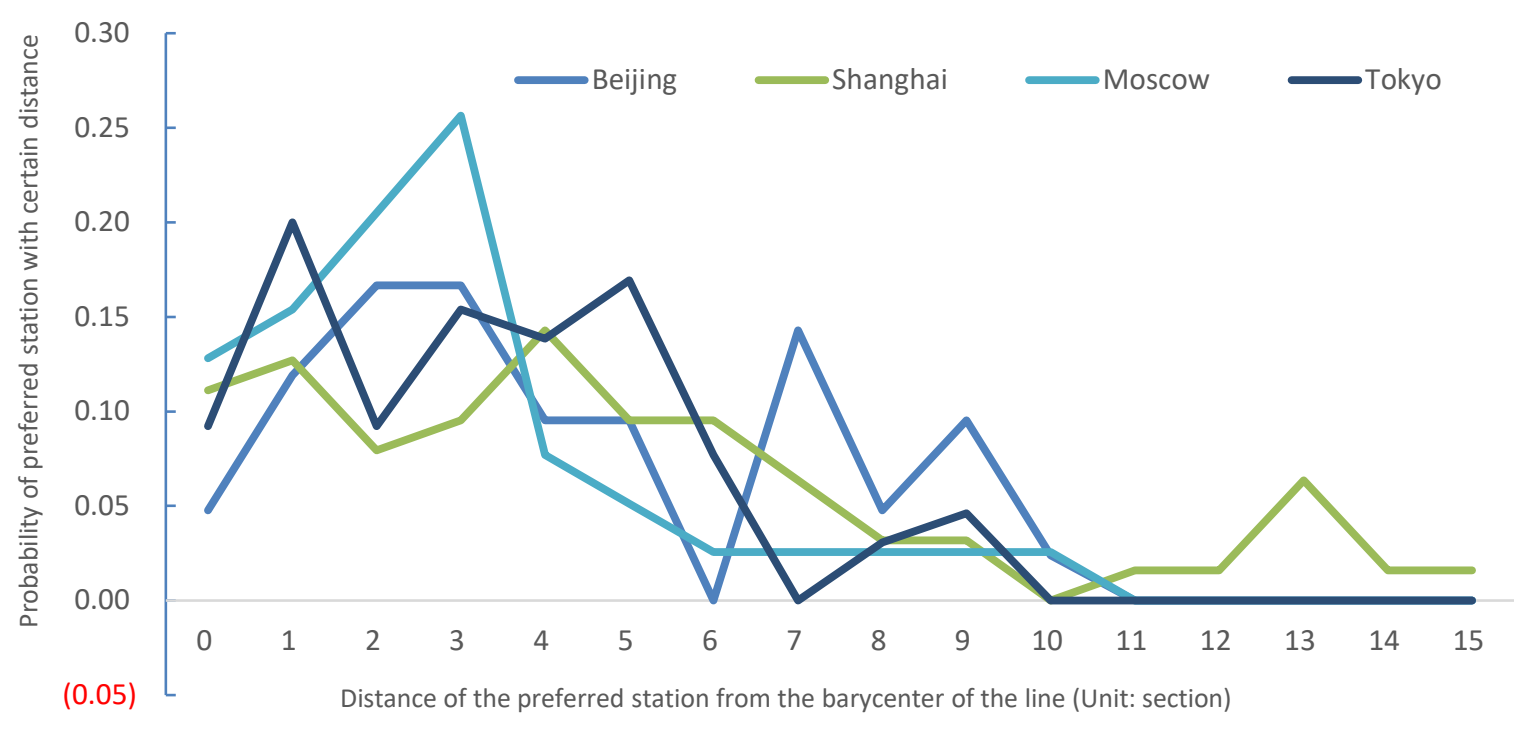

(a). Four subway networks 


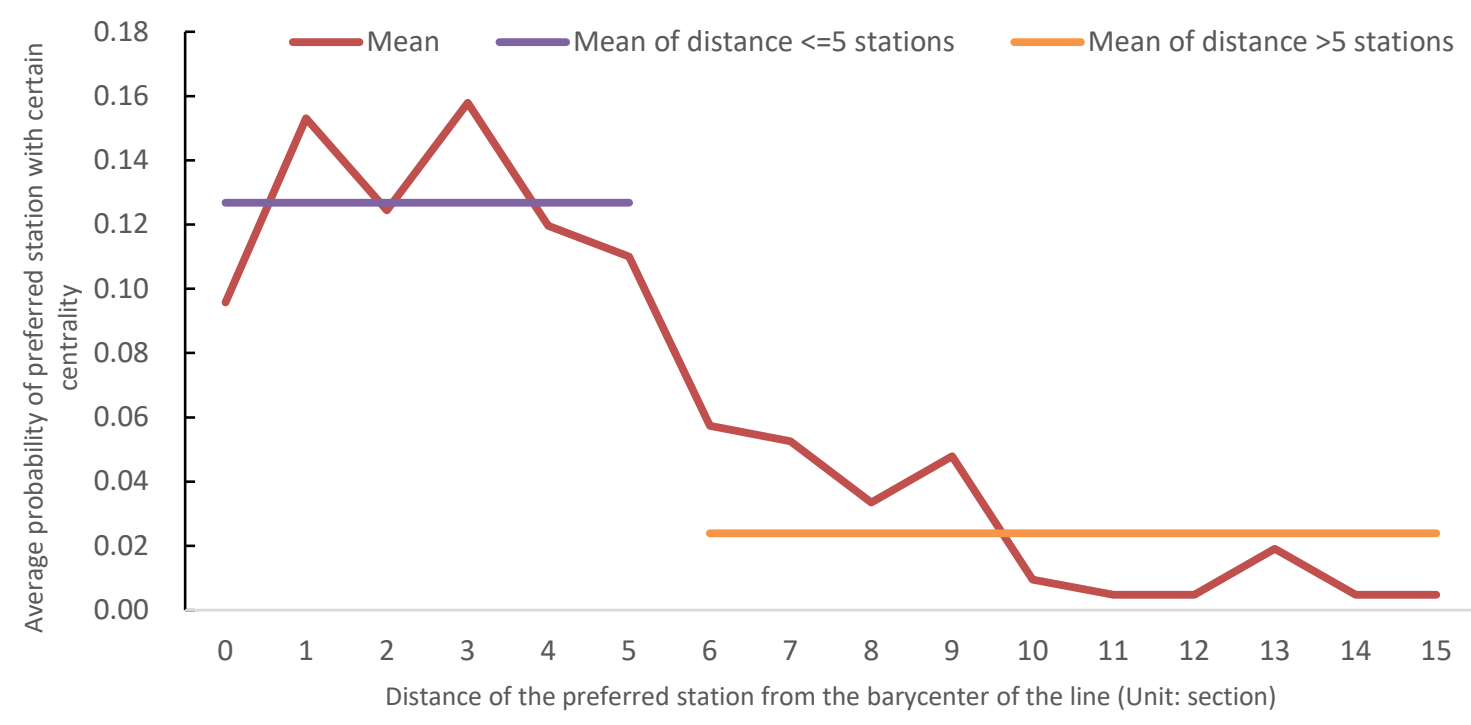

(b) Average probability of four subway networks

Fig. 2 Proportion of a certain distance from the access station to barycenter of the line

As shown in Fig. 2, a rule exists wherein new lines prefer barycenter nearby stations that exhibit a higher HPA ability. As shown in Fig. 2(a), the station with three stations from the barycenter of the line exhibits the highest probability of being selected as the access point for the new line. However, the station with a distance of 15 stations from the center of the line is least likely to be selected as the access point. As shown in the mean value in Fig. 2(b), 12.68\% station with a distance of less than five stations being selected significantly exceeds than that $2.24 \%$ station with a distance greater than five stations. The bigger subway networks such as London, would prove this hypothesis 'new lines preference to the stations with a higher ability of HPA' more favorably. Smaller subway networks do the opposite. Adding data of London underground (bigger subway network) into the model shows that the probability of new lines selecting stations $<=5$ stations from the barycenter of the line was $12.87 \%$, an increase of $0.19 \%$. When the data of 
Guangzhou metro network (smaller subway network) is added, the probability of new lines selecting stations $<=5$ stations from the barycenter of the line was $12.53 \%$, down by $0.14 \%$.

\section{HPA-driven evolution model}

\subsection{Topological preference dependence mechanism of new lines}

According to the analysis in the Section 2, firstly, new lines tend to access networks by connecting with strong transfer capabilities stations, and stations with high transfer capacity tend to exhibit higher degrees. The BA model is optimal and reflects the preference of the topology. Therefore, to realize the topology attachment preference mechanism of the newly added line while accessing the network, the first step involves selecting an access station based on the BA model. The probability of the BA model's preferred connection $\pi\left(k_{i}\right)$ is calculated as follows:

$$
\Pi\left(k_{i}\right)=\frac{k_{i}}{\sum_{j} k_{j}}
$$

Where $i$ denotes the $i$-th station traversed to, $k_{i}$ denotes the total number of subway lines that pass through station $i$ in the current step, $j$ denotes the station in the network in the current step, and $\sum k_{j}$ denotes the sum of the number of lines passing through all stations in the network.

\subsection{HPA to new lines}

Secondly, the clustering coefficient (CC) used here as a mathematical analytical model for the HPA preference of new lines. CC is the earliest index used to describe the proportion of all neighbor nodes being neighbors. Increasing in the CC make the small community closer [33]. In the subway network, the $\mathrm{CC}$ of this type of a small community where all neighboring stations of a station are also neighbors is used to indicate that the stations exhibit a strong ability to attract more 
individuals. Examples including business activity centers, political activity centers, entertainment centers correspond to a hot-point station with good geographical location and great centrality and indicate a higher intensity of the local connection. A station with higher $\mathrm{CC}$ exhibits better location, and it is more convenient to run back and forth between other stations. Therefore, based on the rule of HPA stations with new line preference links, if a station has a large $\mathrm{CC}$, it will improve the probability of being linked by the new line.

However, a station that's one section away from all its neighboring stations has a different ability to attract new lines with a station that's multi-sections away from all its neighboring stations. The two stations in the subway network that do not need to be transferred are termed as neighboring stations, the two direct neighboring stations are one section away from each other and do not need to pass through other stations on the line to reach. The two indirect stations are multi-sections away from each other and need to pass through other neighboring stations on the line to arrive. Therefore, when applied to the subway network, $\mathrm{CC}$ should reflect the difference between direct adjacent and indirect adjacent neighbors. The CC of subway network proposed by the Ref. [10] reflects the difference of the ability of direct neighboring and indirect neighboring small communities to gather individuals. A direct neighbor denotes that two stations are designated on a line that can be reached without going through other stations on the line, as represented by stations $v_{1}$ and $v_{2}$ in Fig. 3. An indirect neighbor denotes that two stations are designated on a line although they need to pass through other stations on the line to arrive as represented by stations $v_{1}$ and $v_{4}$ shown in Fig. 3. The distance between two neighboring stations is defined as the number of sections $a_{i j}$. The distance between directly adjacent neighboring stations is $a_{i j}=1$. 


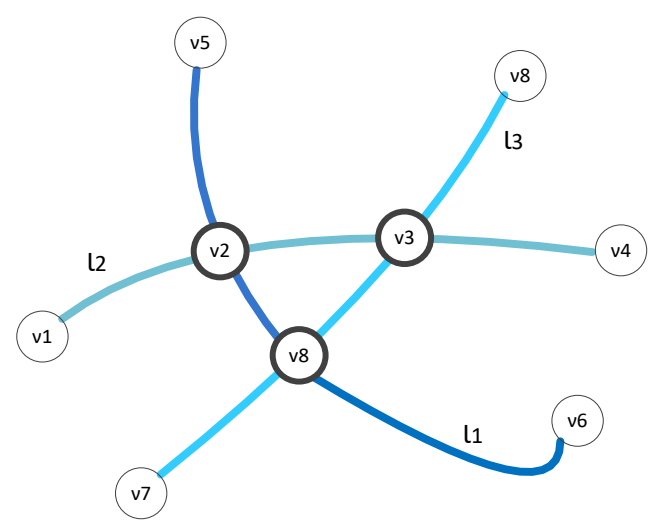

Fig. 3 Sample subway network

Thus, the transformed CC of station is as follows:

$$
\begin{gathered}
c_{i}=2\left(\sum_{k>j, k \in l_{i}, j \notin l_{i}}\left(\frac{1}{a_{i j}}+\frac{1}{a_{i k}}+\frac{1}{a_{j k}}\right)+\sum_{l_{i}}\left(\left(V_{l_{i}}-3\right) \sum_{j \in V_{n e i g h b o r}(i)} \frac{1}{a_{i j}}+\frac{1}{2} \sum_{i, j \in l_{i}} \frac{1}{a_{i j}}\right)\right) / \\
3 V_{\text {neighbor }}(i)\left(V_{\text {neighbor }}-1\right)
\end{gathered}
$$

Where the number of sections between any two stations corresponds to $a_{i j} ; l_{i}$ indicates the route through which station $i$ passes; the number of all neighboring stations of station $i$ is denoted as $V_{\text {neighbor }}(i)$; and the number of stations on line $l_{i}$ is denoted as $V_{\ell_{i}}$. As shown in Eq. (1), the clustering coefficient $c_{i}$ of station $i$ is inversely proportional to $a_{i j}$, i.e., increasing in the closeness of station $i$ to the barycenter of the line will increase $c_{i}$ of station $i$. The physical meaning of the transformed CC is the centrality of a station in the network. In other words, it is the degree of all the neighbor stations which can be reached within one section. Additionally, the transformed CC of station in this research is proportional to the distance from the line barycenter. Therefore, the degree of $\mathrm{CC}$ also depends on how far the station from barycenter is. The attraction probability $\pi\left(C_{n}\right)$ of HPA to the newly added line is as follows:

$$
\prod c_{n}=c_{n} / \sum_{m \in \omega} c_{m}
$$


Where $c_{m}$ denotes the clustering coefficient of any station in the set $\omega=\{1,2,3 \ldots \ldots \mathrm{m}\}$; $\pi\left(c_{n}\right)$ denotes the probability be selected of station $\mathrm{n}$ based on the $c_{n}$ of each station within the set $\omega=\{1,2,3 \ldots \ldots \mathrm{m}\}$.

\subsection{Random walk mechanism in a specified direction}

These two ingredients, preferential attachment and growth, are the core contents of scale-free network evolution [34]. After choosing the station to which a new line is connected by BA and HPA, it is necessary to extend the access station into a new line which contains the designed number of stations. Stations on the new line are not set up randomly, but are limited by many factors. Most subway lines exhibit irregular forms constrained by the planning of land use, passenger attraction, topography, environment, and other factors. However, those lines can be classified into several basic types [16]. Typically, the highest density of travel in urban areas is concentrated in diametrical directions converging on the city center area. Circle or ring lines provide a direct connection among numerous medium-to-high density areas around city center and

enable several suburb-to-suburb trips to use a diametrical-circle-diametrical path. Branches provide direct service from the trunk (see Fig. 4).

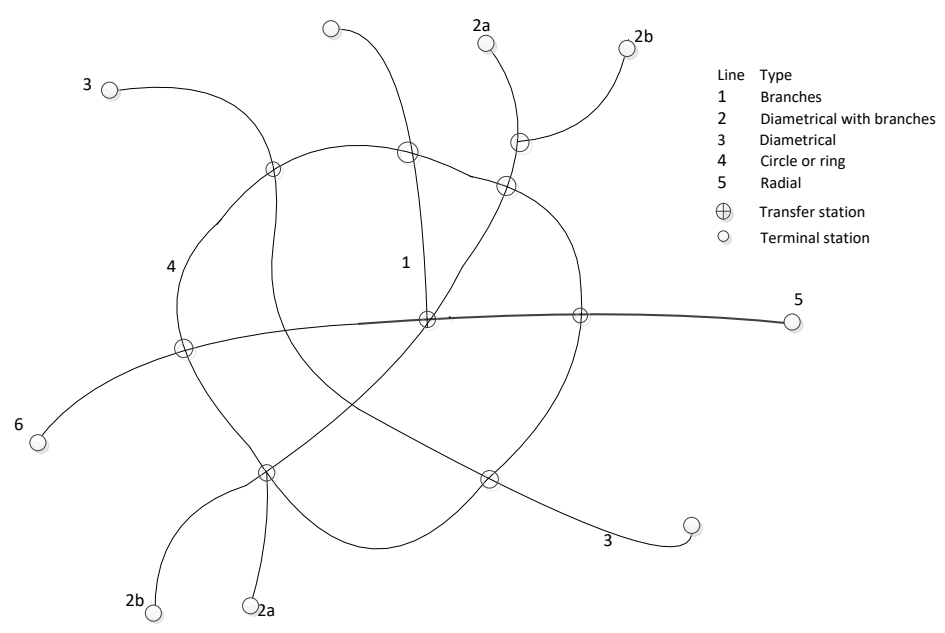


Fig.4 Geometric forms of subway lines

Thus, we defined three walk directions of lines to generate stations that include orthogonal direction, radial direction, and ring direction. Both orthogonal direction and radial direction walks are used to generate stations on diametrical lines. In the radial direction walk, new stations should be generated randomly in a non-vertical orientation of the old station (Fig.5(a)). Conversely, new stations should be created randomly in a vertical orientation of the old station in the orthogonal direction walk (Fig.5(b)). Stations on branch lines can only be generated randomly in the radial direction (Fig.5(c)). In the ring direction walk, new stations should be produced clockwise randomly within a radius(Fig.5(d)).
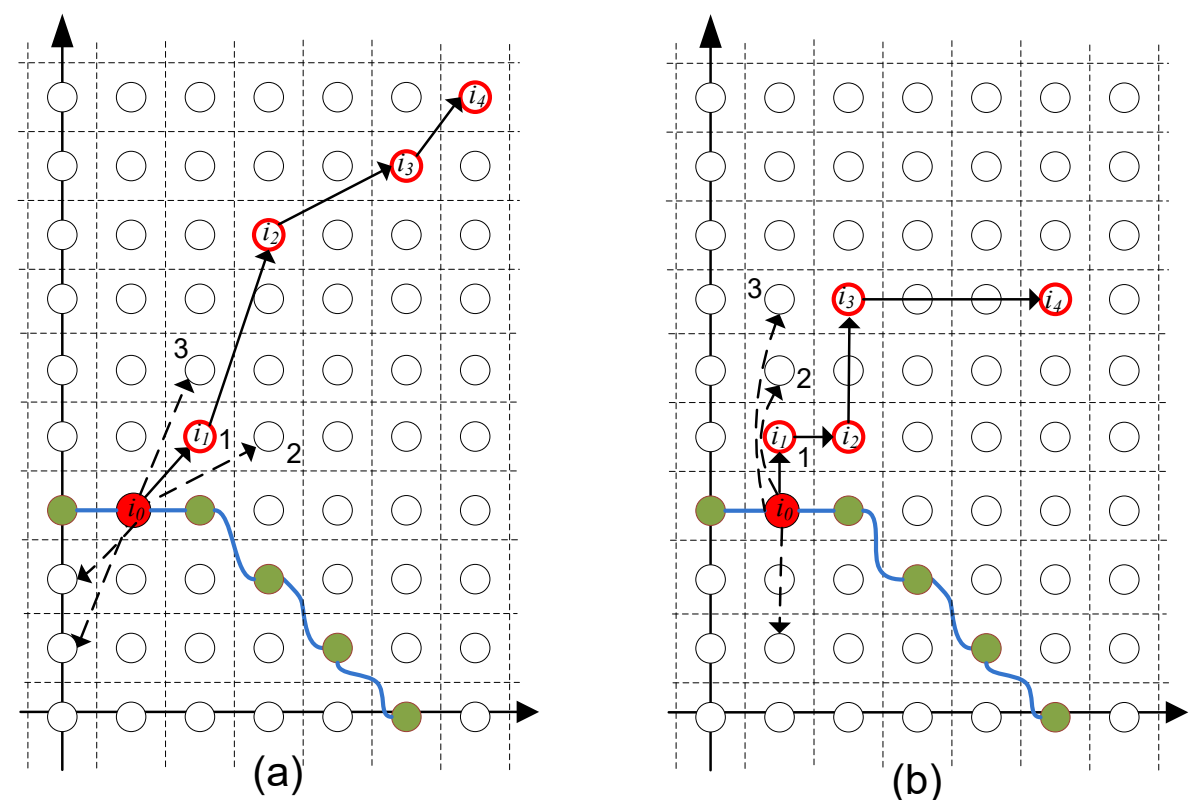

(b) 


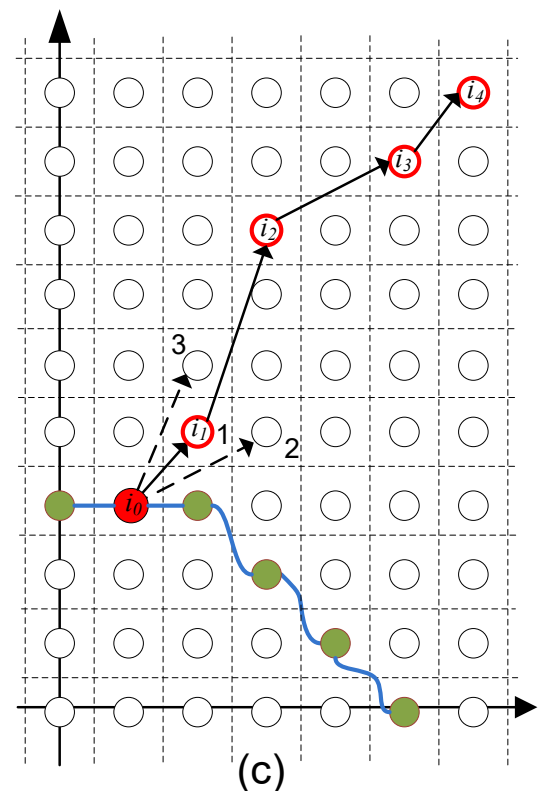

(c)

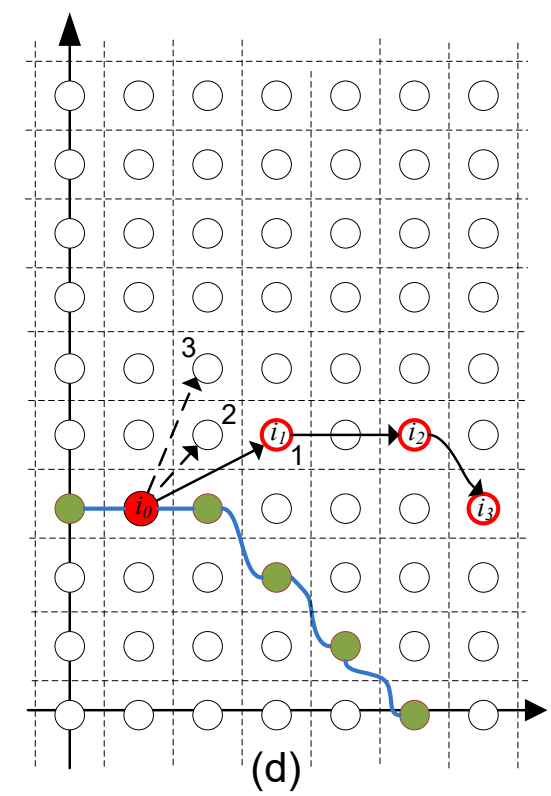

(d)

Fig. 5 Stations generated on specified direction (a) generated diametrical line on radial direction (b) generated diametrical line on orthogonal direction (c) generated branch on radial direction (d) generated circle line on ring direction

\section{Evolution process}

In order to test the effectiveness of the HPA model through simulating the evolution of the subway network, this study defines the continuous evolution rules of the HPA subway network. Given the number of lines $l$ included in the subway network, number of stations $m$ owned by each diametrical line, number of stations owned by branch line $1 / 2 m$, radius of the ring line $r$; orthogonal line proportional control parameter control parameter $a$; radioactive line proportional control parameter $b$, branch line proportional control parameter $c$, and circle line proportional control parameter $d$, the evolution of the HPA subway network is as follows: 
Step $1 \quad t=0$ :

Generate a $(x, y)$ grid commencing at the center of the grid to generate the initial line $l_{0}$;

Step $2 \mathrm{t}=1$ :

Generate a new line $l_{1}$. Calculate $\pi\left(k_{i}\right)$ of all stations on the initial line $l_{0}$, select station $i$ based on the BA model (formula 1).

Step $3 t=2$ :

Because of the low probability of the BA model being selected to the transfer station; the station's line degree does not exhibit a significantly ability to dominate. Therefore, if $\pi\left(k_{i}\right)$ of station $i$ is selected as the highest on the line, then the station is selected as the access point of the newly added line $l_{1}$.

If there exists station(s) that exhibits a line degree greater or equal to $\pi\left(k_{i}\right)$ of station $i$ selected by the BA model, then we obtain the set $\omega=\{1,2,3 \ldots \ldots \mathrm{m}\}$ of all stations equal to or greater than the probability and proceed to the HPA selection. In the set of stations $\omega=$ $\{1,2,3 \ldots \ldots \mathrm{m}\}$ with the same or greater probability $\pi\left(k_{i}\right)$, the CC of each station is calculated. Then, using the HPA model (Formula 3) to perform a second step selection after BA selection, finally obtain the first access point $n$ for the new line $l_{1}$ to access the network, and then proceed to the next step;

Step $4 t=3$ :

After completing the connection between the new line $l_{1}$ and current subway network, stations on the new line would be generated to the designed station number in this step. Starting 
from station $i_{0}$, randomly generate next station $i_{1}$ within one step to three-step neighbor grid. As shown in Fig. 5(a), there are three choices to generate station $i_{1}$ in position 1, 2, and 3 in radial direction. If the grid is occupied by an old station (green color node), connect to that old station. Then start from $i_{1}$, repeat the random walk process in the specified direction until the station number reached to the designed number. There are $m$ stations on each diametrical line, $1 / 2 m$ stations on each branch. Stations on a diametrical line are generated from both sides of station $i_{0}$, but stations on branch and circle line are only generated from one side of station $i_{0}$. Station generation on each circle line should be end till the last station is the start station $i_{0}$.

Step $5 \mathrm{t}=4$ :

Repeat Step 2, 3, and 4, till the number of orthogonal lines reach to $a \times l$, number of radioactive lines reach to $b \times l$, number of branches reach to $c \times l$, and number of circles reach to $d \times l$.

The difference between HPA evolution and BA evolution process is that all the step from 1 to 5 are included in HPA evolution process, but Step 2 is excluded in BA evolution process.

\section{Numerical simulation of the evolution model}

\subsection{Generation of a network}

The mechanism rule for a new line to access a network is identical for different types of topological structures, radial networks, radial/circumferential networks, grid networks, grid /circumferential networks, and ubiquitous networks. However, the walk rules differ from the direction of each type of topological structure. Therefore, we only select a type of topology 
structure of the subway network for simulation to verify the validity of the HPA evolution model. The Moscow subway network corresponds to a typical topological structure, namely a ring with radial type and branch-type lines. The HPA model and BA model are used to simulate the Moscow subway network. With respect to the two different evolution models, only the preference selection rule is different with identical random walk rules in the specified direction.

In the parameter setting, there are 1 ring-shaped line, 8 radial type lines, and 3 branch-type lines, and each line contains 17 stations. The number of trials was determined by using the mean as a criterion. The relationship between the number of test repetitions and number of stations, number of sections, and number of transfer stations of the generated subway network model are shown in Figs. 6, 7, and 8, respectively.

As shown in Figs. $6-8$, the number of stations, sections, and transfer stations significantly fluctuate when the number of trials is less than 60 times. When the number of tests increases to greater than 60 times, the mean curves of the three eigenvalues tend to be stable, and the curve fluctuation is small. The yellow line in the figure denotes the mean line of 100 repeated tests. As shown in the figures 6-8, the result is close to the mean when the number of repetitions reaches 80. The results are slightly improved when the number of repetitions approachs to 100 . Therefore, we use the results for the number of repetitions corresponding to 100 as the basis for analysis. 

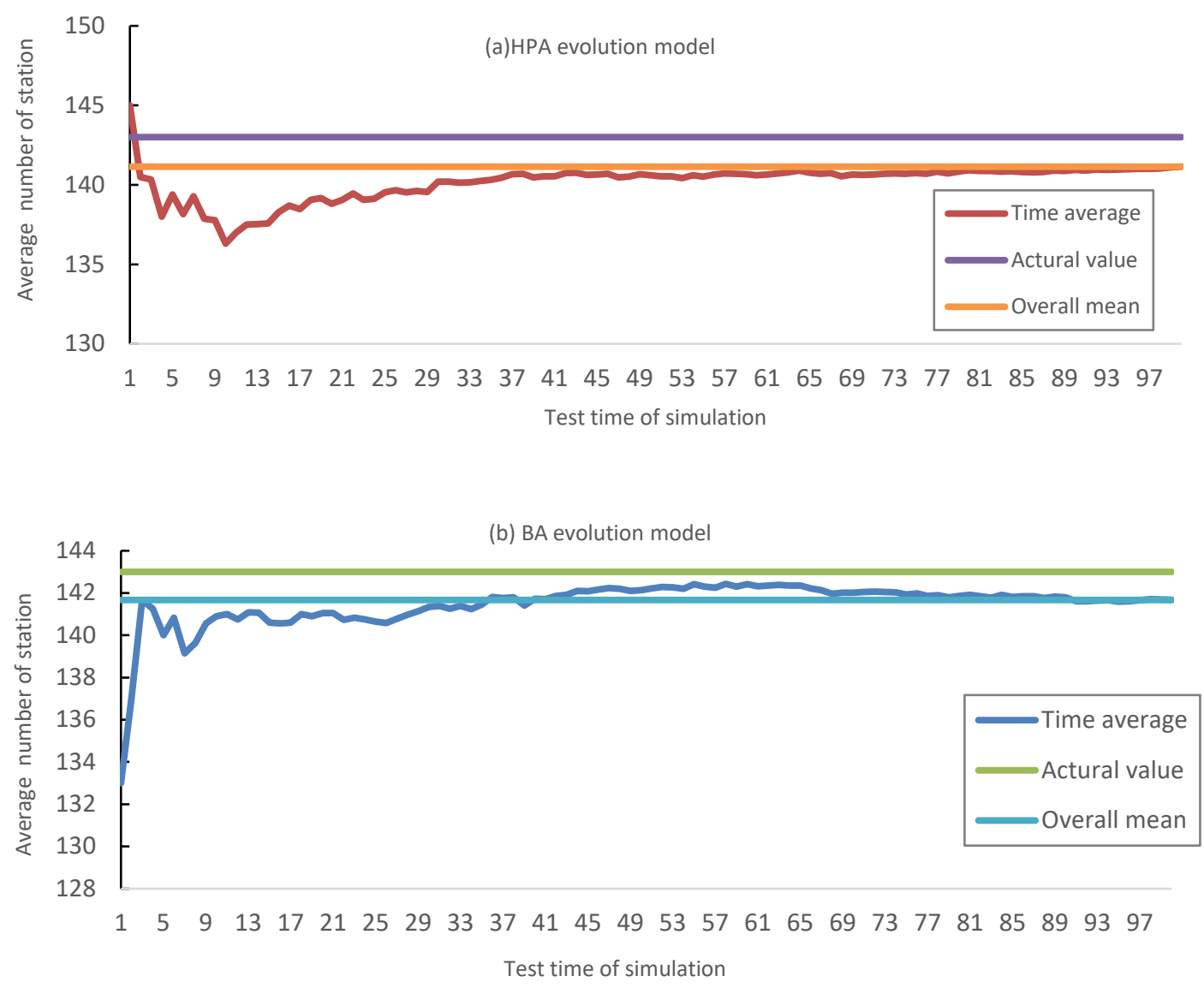

Fig. 6 Number of test repetitions and the curve showing the average number of subway stations

(a)HPA evolution model (b)BA evolution model

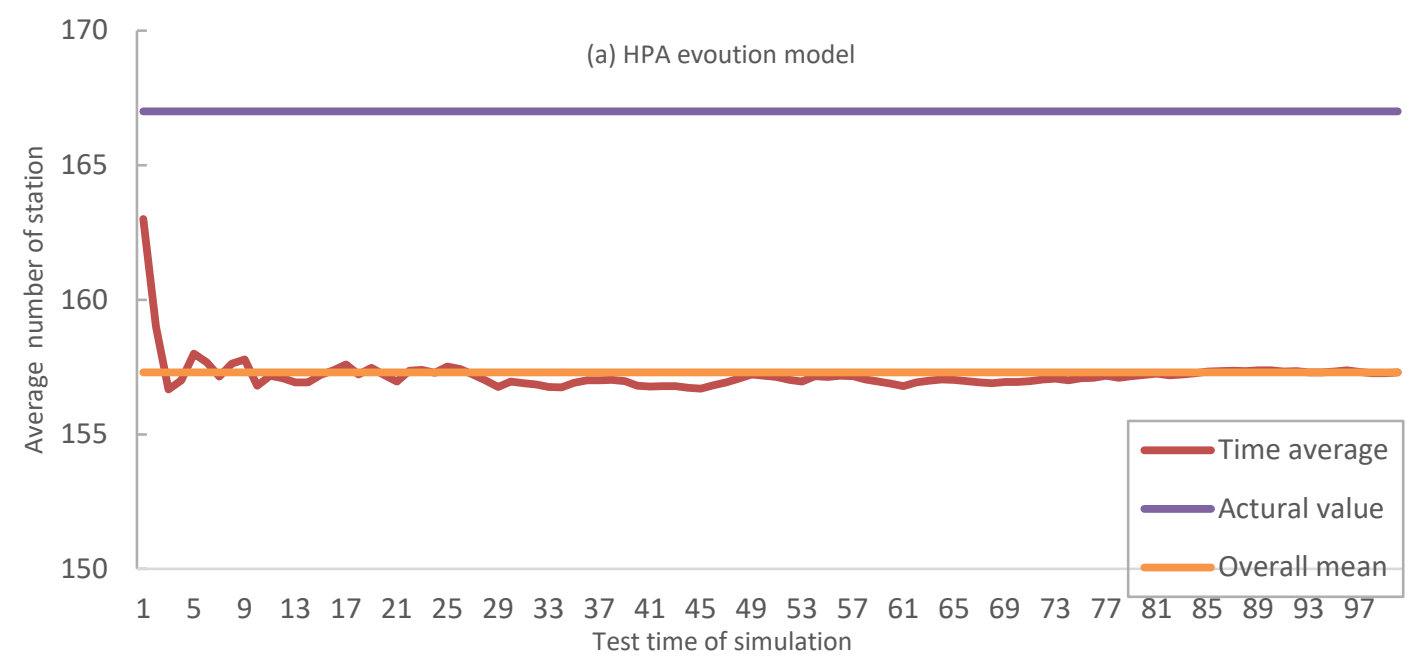




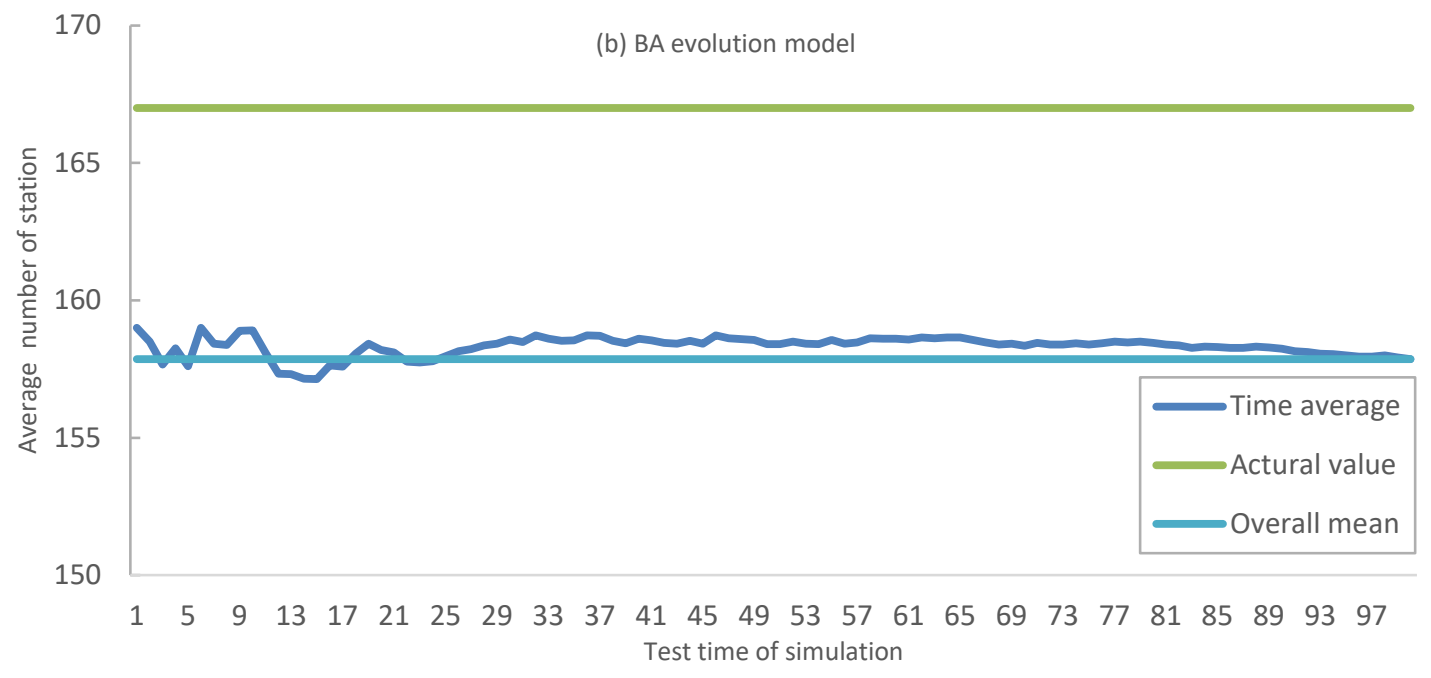

Fig. 7 Number of test repetitions and the curve showing the average number of subway sections

(a)HPA evolution model (b)BA evolution model
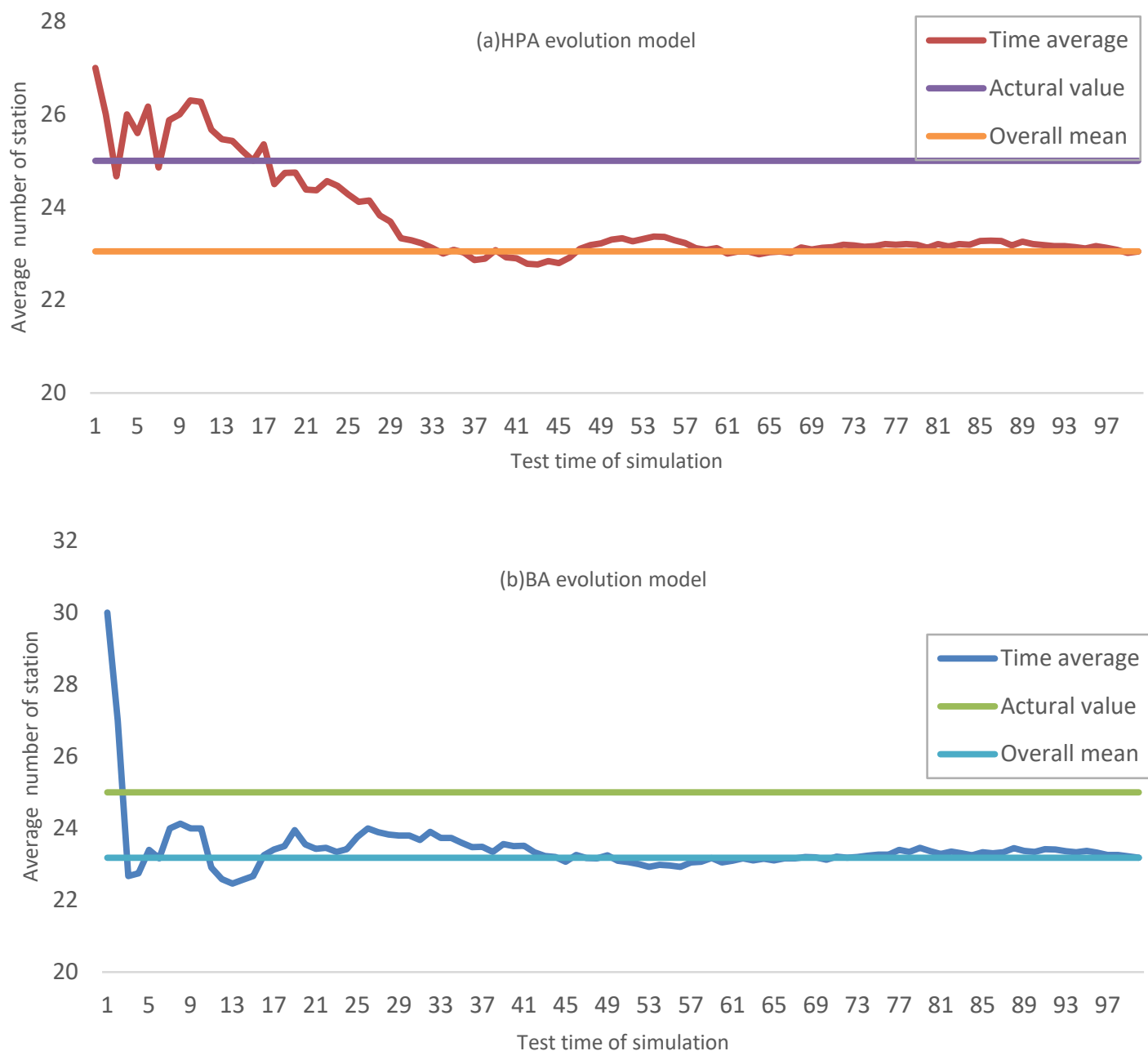

Fig. 8 Number of test repetitions and the curve showing the average number of subway transfer 
stations (a)HPA evolution model (b)BA evolution model

\subsection{Model reliability and validity test}

The HPA and BA evolution model experiments via Myeclipse simulation platform were each repeated 100 times. The average value $(\mathrm{AV})$, standard deviation $(\mathrm{SD})$, and root mean square error (RMSE) of the generated subway network are shown in Table 1.

Table 1 Comparison of results of the test with 100 repetitions

\begin{tabular}{|c|c|c|c|c|c|c|c|}
\hline \multirow{2}{*}{ Eigenvalues } & \multirow[t]{2}{*}{ True value } & \multicolumn{3}{|c|}{ HPA model } & \multicolumn{3}{|c|}{ BA model } \\
\hline & & AV & SD & RMSE & AV & $\mathrm{SD}$ & RMSE \\
\hline stations & 143 & 141 & 5.20 & 6.18 & 141 & 5.76 & 5.89 \\
\hline sections & 167 & 157 & 4.24 & 10.58 & 157 & 4.62 & 10.23 \\
\hline Transfer stations & 25 & 23 & 4.41 & 4.48 & 23 & 4.79 & 5.1 \\
\hline
\end{tabular}

As shown in Table 1, the standard deviation (SD) of the number of stations, the number of sections, and the number of transfer stations generated by the HPA model are 5.20, 4.24, and 4.41, respectively, and are slightly lower than those of the BA model that are 5.76, 4.62, and 4.79, respectively. Therefore, the stability of the HPA model is relatively higher, and the degree of dispersion of the test results is relatively lower. Although the number of stations and the number of sections of the subway network generated based on the HPA model are 6.18 and 10.58, respectively, and this slightly exceeds that of the BA model, the RMSE of the subway network generated by the BA model correspond to 5.89 and 10.23, respectively, the RMSE of the number 
of transfer stations are 4.48, which is significantly lower than 5.1 for the BA model. In the subway network, the number of transfer stations are the most important factor that affects network heterogeneity and connectivity. Nevertheless, increasing or decreasing one non-transfer station does not significantly affect the dynamic characteristics of the network. Thus, from the perspective of model stability and effectiveness, the evolution mechanism based on the HPA evolution model exhibits a better performance than that of the BA evolution model.

\section{Discussion}

First, the fluctuations in the new line access points of the subway network generated by the HPA evolution model and BA evolution model are analyzed. The horizontal axis $\mathrm{x}$ in Fig. 9 denotes the distance of the access point from the barycenter of the line, vertical axis y denotes the probability value of the access point for a certain distance, and the colored line denotes the number of experiments. As shown in Fig. 9, the access point significantly fluctuates when the number of experiments is less than 40 . When the number of trials corresponds to 80 , the fluctuation of the access point decreases, and the trend of 80,90 , and 100 times changes the minor. The average selection probability of the access points that were tested 100 times was credible. 

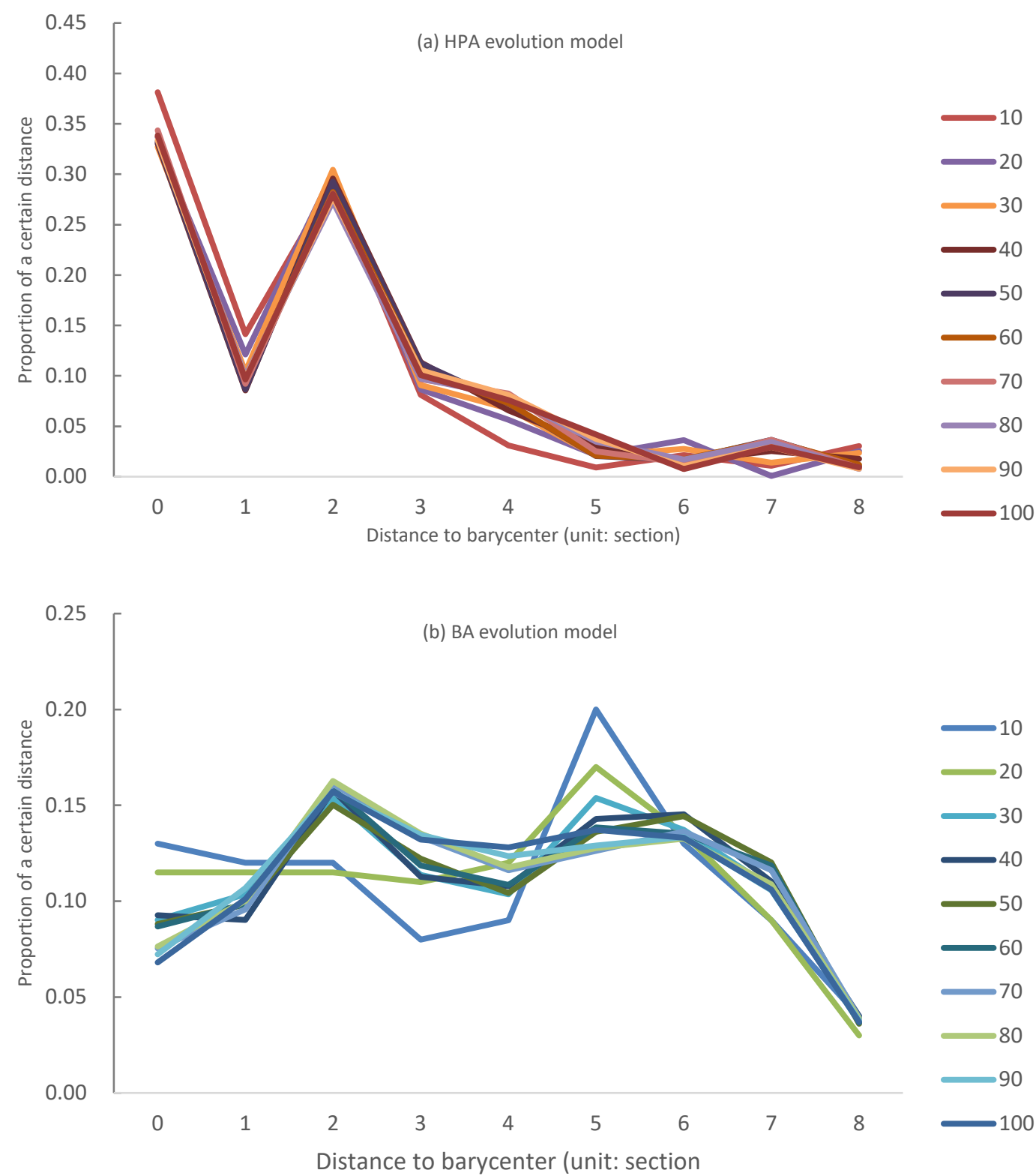

Fig. 9 Number of test repetitions and probability of the selection point curve

(a) HPA evolution model (b)BA evolution model

The experiment is repeated 100 times, and the average selection probability of the access point of the HPA model and BA model is compared as shown in Table 2. In the HPA model, the probability of selecting the barycenter station as the access point is $33.84 \%$, and this exceeds that of the BA model (6.81\%). The probability of selecting the distance within $0 \sim 3$ sections exhibits a clear downward trend, and this is consistent with the results of Equation 2. In the BA model, the 
choice of the access point does not exhibit a significant correlation with the location of the station, and the randomness is high. Two peaks of selection probability are observed with 2 and 5 stations away from the barycenter. The probability of choosing distance 1 (1 section) away from the barycenter exceeds that of the HPA model although the probability of selecting distance 2 (2 sectios) is less than the HPA model. The probability of selecting the farthest station from the city center is $3.70 \%$, and this exceeds that of the HPA model $(0.91 \%)$. The average probability of access distance within 4 station obtained by HPA is $20.41 \%$, which is closer to 18.59 of Moscow subway network than $11.46 \%$ obtained by BA.

Table 2 Average selection probability of the access point

\begin{tabular}{rrrrrrrrrr}
\hline distance & 0 & 1 & 2 & 3 & 4 & 5 & 6 & 7 & 8 \\
\hline HPA model & station & station & stations & stations & stations & stations & stations & stations & stations \\
\hline BA model & $6.84 \%$ & $9.65 \%$ & $28.07 \%$ & $10.08 \%$ & $7.58 \%$ & $4.17 \%$ & $0.75 \%$ & $2.95 \%$ & $0.91 \%$ \\
\hline
\end{tabular}

Subsequently, we compare the evolution subway network with the real subway network via the cumulative probability of access distance to barycenter (see Fig. 10). As shown in the figure, the cave of HPA is closer to the real subway network access process. 


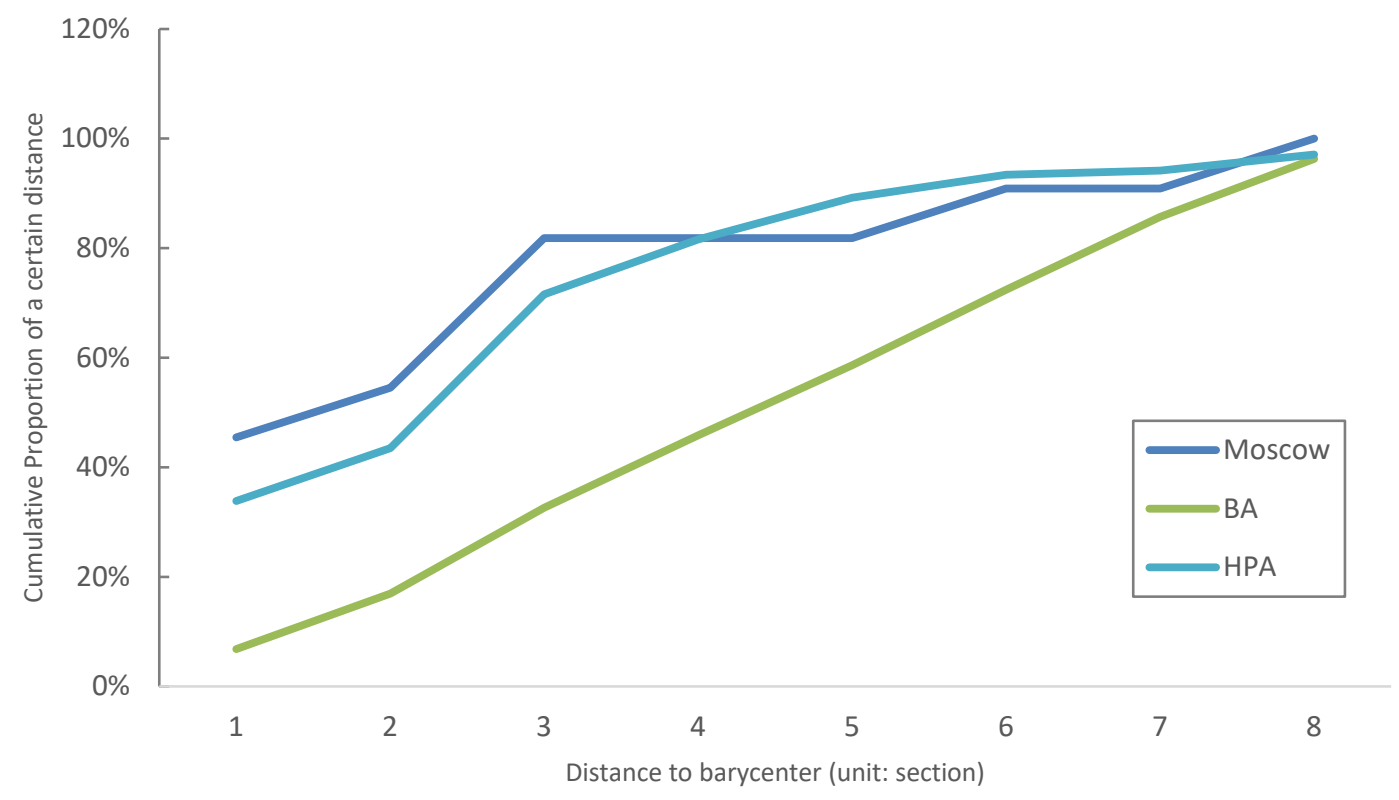

Fig. 10 Cumulative probability of access distance to the barycenter of the line

The above results indicate that the HPA model exhibits a higher probability of being closer to the barycenter of the line (within 5 stations), i.e., the transfer station is more likely to be located closer to the city center and the evolution mechanism is more likely. This reflects the attraction characteristics of the geographical location attribute of the old station in the urban space to the new line and is more in agreement with the dynamic evolution mechanism of the actual subway network.

In order to further verify the effectiveness of the HPA model for subway network simulation, the study selects the seven characteristics of the number of stations (NS), number of transfer stations (NTS), number of connections (NC), scale factor(SF), minimum transfer(MT), CC, and 
maximum connected path (MCP) and compares the same with the results of the BA model. The details are given in Table 3.

Table 3 Comparison of eigenvalues between HPA model and BA model with 100 tests

\begin{tabular}{cccccccc}
\hline Model & NS & NTS & NC & SF & MT & CC & MCP \\
\hline Moscow & 143 & 25 & 167 & -2.983 & 0.9956 & 0.0056 & 20306 \\
HPA model & 141 & 23 & 157 & -2.8794 & 1.4595 & 0.0043 & 19952 \\
BA model & 141 & 23 & 157 & -2.8787 & 1.4669 & 0.0039 & 19831 \\
\hline
\end{tabular}

As shown in Table 3, the metro network generated by the HPA model exhibits a scale factor, an average minimum transfer number, a clustering coefficient, and a maximum connectivity path feature value closer to the original value. This indicates that the HPA model is more capable of realizing the evolution mechanism of the subway network.

\section{Conclusion}

The subway topology network represents the basis of the interaction between subsystems.

The subway network evolution mechanism accurately reflects the actual subway network, and directly affects studies on the dynamic behavior of subsystems. The HPA evolution rules proposed in the study describe the evolution mechanism of the actual subway network by increasing the attraction of the station's geographical center degree to the new line and random walk of the new line in the specified direction. The following conclusions were obtained:

(1) With respect to the preferential mechanism of the subway network, an evolution model 
that reflects the attraction of the station's geographical center degree to the new route is required, and the existing evolution model fails to fully consider the physical orbital construction process in the subway network. Long-distance construction of subway track typically entails a high cost. The HPA evolution model proposed in the study solves the shortcomings of existing results in subway network evolution and can be used to guide the design of subway topology.

(2) Although the number of stations and the number of sections of the subway network generated by the HPA evolution model do not exhibit evident advantages, they are closer to the real network in terms of the transfer station, and this significantly exceeds that of the BA model. The transfer station in the subway network directly affects the degree of heterogeneity of the network which corresponds to the key factor that affects the dynamic characteristics of the subway network.

(3) Based on the centrality of the transfer station in the subway network, the transfer station in the subway network generated by the HPA evolution model exhibits a higher probability of being closer to the barycenter of line, and this is more in line with the dynamic evolutionary mechanism of the real subway network.

(4) In terms of parameters including the SF, MT, and CC, the fitting degree based on the HPA evolution model exceeds that of the BA model. However, the evolution rule requires further improvement to any real application. 


\section{Acknowledgements}

The study was supported by the Natural Science Foundation of Beijing Municipality (L181009 and 9194028), Humanities and Social Science Fund of Ministry of Education of China (18YJC630193), and Fundamental Research Funds for the Central Universities of University of Science and Technology Beijing (2015M570121). The authors would like to acknowledge the experts who participated in the study and those who provide suggestions.

\section{References}

1. V. Latora, M. Marchiori, Efficient behavior of small-world networks[J]. Phys. Rev. Lett.,. 87(19) (2001) 198701. https://doi.org/10.1103/physrevlett.87.198701

2. J. Sienkiewicz, J. A. Holyst, Statistical analysis of 22 public transport networks in Poland, Physical Review E: Statistical, Phys Rev E Stat Nonlin Soft Matter Phys., 72(4 Pt 2) (2005) 046127. https://doi.org/10.1103/physreve.72.046127

3. von Ferber C, Holovatch T, Holovatch Y, Palchykov V. 2009. Public transport networks: empirical analysis and modeling $[\mathrm{J}] . \quad$ EPJB, 68 68(2) https://doi.org/10.1140/epjb/e2009-00090-x

4. S. Wandelt, X. Sun, Evolution of the international air transportation country network from 2002 to 2013, Transportation Res. Part E: Logistics Transportation Rev., 82(2015) 55-78. https://doi.org/10.1016/j.tre.2015.08.002

5. Z. Yang, X. Chen, Evolution assessment of Shanghai Urban Rail Transit Network, Physica A, 503(2018) 1263-1274. https://doi.org/10.1061/41039(345)506 
6. X Zhou, L Ping, Research on Mass Transit Network Growing and the Determinants of Network Formation, Traffic \& Transportation, $0(z 1) \quad(2016) \quad 148-151 . \quad$ (in Chinese) doi:10.3969/j.issn.1671-3400.2016.z1.033

7. Y. Sui, F. Shao, et al., Space evolution model and empirical analysis of an urban public transport network, Physica A, 391 (14) (2012) 3708-3717. https://doi.org/10.1016/j.physa.2012.01.011

8. A. Huang, G. Zang, et al., Comparative empirical analysis of flow-weighted transit route networks in R-space and evolution modeling, Int. J Mod. Phys. B, 31 (2017) (175008712). https://doi.org/10.1142/s0217979217500874

9. H. Chang, X. Xu, et al., A manipulator game model of urban public traffic network, Physica A, 416(2014) 378-385. https://doi.org/10.1016/j.physa.2014.09.015

10. Z. Wang, G. Su, et al., Information transfer efficiency based small-world assessment methodology for metro networks, J. Tsinghua University $56 \quad$ (4) (2016) 411-416. DOI : 10.16511/j.cnki.qhdxxb.2016.24.012

11. M. Cai, Y. Cui, et al., Analysis and evaluation of the entropy indices of a static network structure, Sci. Rep-UK, 7 (1) (2017) 9340-10. https://doi.org/10.1038/s41598-017-09475-9

12. Z. Wang, A. P. C. Chan, et al., Recent Advances in Modeling the Vulnerability of Transportation Networks, J $\quad$ Infrastructure $\quad$ Syst., $\quad$ 21(2015) (060140022). https://doi.org/10.1061/(asce)is.1943-555x.0000232

13. P. Sen, S. Dasgupta, et al., Small-world properties of the Indian railway network, Phys Rev E Stat Nonlin Soft Matter Phys., $\quad 67 \quad(3 \quad$ Pt $\quad 2) \quad$ (2003) 036106. 
https://doi.org/10.1103/physreve.67.036106

14. P. Angeloudis, D. Fisk, Large subway systems as complex networks, Physica A, 367(2006) 553-558. https://doi.org/10.1016/j.physa.2005.11.007

15. G. Menichetti, D. Remondini, et al., Weighted multiplex networks, PloS one 9 (6) (2014) e97857. https://doi.org/10.1371/journal.pone.0097857

16. V R. Vuchic, Urban transit: operations, planning, and economics[M]. John Wiley \& Sons. Hoboken, New Jersey, United States 2017. https://doi.org/10.1111/j.1467-9787.2006.00453 2.x

17. J. Zhang, M. Zhao, et al., Networked characteristics of the urban rail transit networks, Physica A, 392 (6) (2013) 1538-1546. https://doi.org/10.1016/i.physa.2012.11.036

18. C. Roth, S. M. Kang, et al., A long-time limit for world subway networks, J R Soc. Interface, 9 (75) (2012) 2540-50. https://doi.org/10.1098/rsif.2012.0259

19. Y. Wang, J. Wang, et al., An evolution model of microblog user relationship networks based on complex network theory, Acta Phys. Sin-CH ED, 63 (2014) (20890220). doi:10.7498/aps.63.208902

20. B. Leng, X. Zhao, et al., Evaluating the evolution of subway networks: Evidence from Beijing Subway Network, EPL (Europhysics Letters) $105 \quad$ (5) (2014) 58004. https://doi.org/10.1209/0295-5075/105/58004

21. W. Du, X. Zhou, et al., Analysis of the Chinese Airline Network as multi-layer networks, Transportation Res. Part E: Logistics Transportation Rev., 89(2016) 108-116. https://doi.org/10.1016/j.tre.2016.03.009 
22. J. Zhang, S. Wang, et al., Characteristics on hub networks of urban rail transit networks, Physica A, 447(2016) 502-507. https://doi.org/10.1016/j.physa.2015.12.060

23. M. Zanin, X. Sun, et al., Studying the Topology of Transportation Systems through Complex Networks: Handle with Care, J Adv. Transport., 2018(2018) 1-17. https://doi.org/10.1155/2018/3156137

24. J. Qian, D. Han, et al., Dynamical evolution of complex airline system, Acta Phys. Sin-CH ED, 60(2011) (0989019). doi:10.7498/aps.60.098901

25. Y. Ding, Z. Ding, et al., The network model of urban subway networks with community structure, Acta Phys. Sin-CH ED, 62 (9) (2013) (0989019). doi:10.7498/aps.62.098901

26. Z. Wang, Z. Liang, et al., Scale-free analysis of subway network, Journal of Southeast University (Natural Science Edition) 43 (4) (2013) 895-9. doi:10.3969/j.issn.1001-0505.2013.04.041

27. S. Derrible, C. Kennedy, Applications of Graph Theory and Network Science to Transit Network Design, $\quad$ Transport $\quad$ Rev., $\quad 31 \quad$ 495-519. https://doi.org/10.1080/01441647.2010.543709

28. C. von Ferber, Y. Holovatch, Fractal transit networks: Self-avoiding walks and Lévy flights, EPJ ST 216 (1) (2013) 49-55. https://doi.org/10.1140/epjst/e2013-01728-0

29. X. Zhou, L. Zhi, Research on Mass Transit Network Growing and the Determinants of Network Formation, Traffic\&Transportation.2016(01) (2016) 148-151.(in Chinese) $\underline{\text { doi:10.3969/j.issn.1671-3400.2016.z1.033 }}$

30. Y. Ding, J. Meng, et al., Statistical properties of random clique networks, Front. Phy. 12 (2017) 
(1289095). https://doi.org/10.1007/s11467-017-0682-x

31. Y. Chen, N. Li, et al., A study on some urban bus transport networks, Physica A, 376(2007) 747-754. https://doi.org/10.1016/j.physa.2006.10.071

32. T. Shanmukhappa, I. W. Ho, et al., Spatial analysis of bus transport networks using network theory, Physica A, 502(2018) 295-314. https://doi.org/10.1016/j.physa.2018.02.111

33. D J. Watts. A simple model of global cascades on random networks[J]. Proceedings of the National Academy of Sciences of the United States of America, 99(9) (2002) 5766-5771. https://doi.org/10.1515/9781400841356.497

34. R. Albert, A. L. Barabasi, Statistical mechanics of complex networks, Rev. Mod. Phys., 74 (1) (2002) 47-97. https://doi.org/10.1007/978-3-540-44943-0 1 